\title{
On location in a half-plane of zeros of perturbed first order entire functions
}

\author{
Michael Gil'
}

Abstract. We consider the entire functions

$$
\begin{gathered}
h(z)=\sum_{k=0}^{\infty} \frac{a_{k} z^{k}}{k !} \text { and } \tilde{h}(z)=\sum_{k=0}^{\infty} \frac{\tilde{a}_{k} z^{k}}{k !} \\
\left(a_{0}=\tilde{a}_{0}=1 ; z, a_{k}, \tilde{a}_{k} \in \mathbf{C}, k=1,2, \ldots\right), \text { provided } \\
\sum_{k=0}^{\infty}\left|a_{k}\right|^{2}<\infty, \sum_{k=0}^{\infty}\left|\tilde{a}_{k}\right|^{2}<\infty
\end{gathered}
$$

and all the zeros of $h(z)$ are in a half-plane. We investigate the following problem: how small should be the quantity $q:=\left(\sum_{k=1}^{\infty}\left|a_{k}-\tilde{a}_{k}\right|^{2}\right)^{1 / 2}$ in order to all the zeros of $\tilde{h}(z)$ lie in the same half-plane?

\section{IntRodUCtion AND STATEMENT OF THE MAIN RESUlt}

Consider the entire functions

$$
h(z)=\sum_{k=0}^{\infty} \frac{a_{k} z^{k}}{k !} \quad \text { and } \quad \tilde{h}(z)=\sum_{k=0}^{\infty} \frac{\tilde{a}_{k} z^{k}}{k !}
$$

$\left(a_{0}=\tilde{a}_{0}=1 ; z, a_{k}, \tilde{a}_{k} \in \mathbb{C}, k=1,2, \ldots\right)$ under the conditions

$$
\sum_{k=0}^{\infty}\left|a_{k}\right|^{2}<\infty \text { and } \sum_{k=0}^{\infty}\left|\tilde{a}_{k}\right|^{2}<\infty .
$$

Any function of the type

$$
\hat{h}(z)=\sum_{k=0}^{\infty} \frac{b_{k} z^{k}}{k !}
$$

with $\left|b_{k}\right| \leq$ const $c_{0}^{k}$ and $c_{0} \geq 1$, can be reduced to the form (1) with condition (2) if we take $z=w / 2 c$.

2010 Mathematics Subject Classification. Primary: 30C15; Secondary: 30D10, 30D20, $30 \mathrm{C} 15$.

Key words and phrases. Entire functions, zeros, perturbations.

Full paper. Received 6 September 2018, revised 21 February 2019, accepted 25 February 2019, available online 25 April 2019. 
Let $z_{k}(f)(k=1,2, \ldots)$ denote the zeros of a function $f$ taken with their multiplicities. We consider the following problem: let

$$
\inf _{k=1,2, \ldots} \Re z_{k}(h)>\gamma \quad(\gamma=\text { const }>0) .
$$

How small should be the quantity

$$
q:=\left(\sum_{k=1}^{\infty}\left|a_{k}-\tilde{a}_{k}\right|^{2}\right)^{1 / 2}
$$

in order to provide the inequality

$$
\inf _{k=1,2, \ldots} \Re z_{k}(\tilde{h})>\gamma ?
$$

The literature on perturbations of the zeros of analytic functions is rather rich. In particular, the results obtained enable us to explore the relations between the zeros of the power series, their partial sums and tails, cf. [5], to estimate the distances between the zeros of entire functions and the zeros of their derivatives, $[2,3,6,8]$. The variation of the zeros of general analytic functions under perturbations was investigated, in particular, by $\mathrm{P}$. Rosenbloom [17]. He has established the perturbation result that provides the existence of a zero of a perturbed function in a given domain. In the case of entire functions the Rosenbloom's results have been refined in [9] (see also [11]). Of course we cannot survey the whole subject here and refer the reader to the just mentioned papers and books, and references given therein. However, to the best of our knowledge the above pointed problem was not not considered in the available literature although it is important, in particular, for localization of the zeros of perturbed functions. Our main tool is the recent norm estimates for solutions of the perturbed Lyapunov equation.

Put

$$
\psi(h):=\left(\sum_{k=1}^{\infty}\left|a_{n}\right|^{2}+\zeta(2)-1\right)^{1 / 2}, \text { where } \zeta(z)=\sum_{k=1}^{\infty} \frac{1}{k^{z}}(\Re z>1)
$$

is the Riemann zeta function. Below we show that condition (3) implies

$$
\sup _{k}\left|\frac{2 \gamma}{z_{k}(h)}-1\right|<1
$$

and therefore

Finally, denote

$$
r(h, \gamma):=1-\sup _{k}\left|\frac{2 \gamma}{z_{k}(h)}-1\right|>0
$$

$$
\xi(h, \gamma):=\left(\sum_{m=0}^{\infty} \frac{(2 \gamma \psi(h))^{m}}{\sqrt{m !} r^{m+1}(h, \gamma)}\right)^{2} .
$$

In Section 4 we suggest estimates for $\psi(h), q$ and $\xi(h, \gamma)$. Now we are in a position to formulate the main result of this paper. 
Theorem 1.1. Let $h(z)$ and $\tilde{h}(z)$ be defined by (1). Let the conditions (2), (3) and

$$
4 \xi(h, \gamma)\left(\gamma^{2} q^{2}+\gamma q(1+2 \gamma \psi(h))\right)<1
$$

be fulfilled. Then inequality (4) is valid.

The proof of this theorem is presented in the next section.

\section{Proof of Theorem 1.1}

Let $\mathbb{C}^{n}$ be the complex $n$-dimension Euclidean space with a scalar product $(.,$.$) and the norm \|\|=.\sqrt{(., .)}$. Denote by $\mathbb{C}^{n \times n}$ the set of $n \times n$-matrices. For an $A \in \mathbb{C}^{n \times n}, \lambda_{k}(A)(k=1, \ldots, n)$ are the eigenvalues taken with the multiplicities, $\sigma(A)$ is the spectrum, $r_{s}(A)=\max _{k}\left|\lambda_{k}(A)\right|$ is the spectral radius, $A^{*}$ is the adjoint one, and $\|A\|$ is the spectral norm: $\|A\|^{2}=r_{s}\left(A^{*} A\right)$; $I$ is the unit $n \times n$-matrix.

For an integer $n>1$, let us consider the polynomials

$$
f_{n}(\lambda)=\sum_{k=0}^{n} \frac{a_{k} \lambda^{n-k}}{k !} \quad \text { and } \quad \tilde{f}_{n}(\lambda)=\sum_{k=0}^{n} \frac{\tilde{a}_{k} \lambda^{n-k}}{k !} .
$$

$f_{n}$ is the characteristic polynomial of the matrix

$$
\left(\begin{array}{ccccc}
-a_{1} & -a_{2} / 2 ! & \cdots & -a_{n-1} /(n-1) ! & -a_{n} / n ! \\
1 & 0 & \cdots & 0 & 0 \\
0 & 1 & \cdots & 0 & 0 \\
\vdots & \vdots & \ddots & \vdots & \vdots \\
0 & 0 & \cdots & 1 & 0
\end{array}\right) .
$$

As it is shown in [11] Lemma 5.2.1, p. 117, this matrix is similar to the following one

$$
F_{n}=\left(\begin{array}{ccccc}
-a_{1} & -a_{2} & \cdots & -a_{n-1} & -a_{n} \\
1 / 2 & 0 & \cdots & 0 & 0 \\
0 & 1 / 3 & \cdots & 0 & 0 \\
\vdots & \vdots & \ddots & \vdots & \vdots \\
0 & 0 & \cdots & 1 / n & 0
\end{array}\right) .
$$

So $z_{k}\left(f_{n}\right)=\lambda_{k}\left(F_{n}\right)(k=1, \ldots, n)$. Similarly, $z_{k}\left(\tilde{f}_{n}\right)=\lambda_{k}\left(\tilde{F}_{n}\right)(k=1, \ldots n)$, where

$$
\tilde{F}_{n}=\left(\begin{array}{ccccc}
-\tilde{a}_{1} & -\tilde{a}_{2} & \cdots & -\tilde{a}_{n-1} & -\tilde{a}_{n} \\
1 / 2 & 0 & \cdots & 0 & 0 \\
0 & 1 / 3 & \cdots & 0 & 0 \\
\vdots & \vdots & \ddots & \vdots & \vdots \\
0 & 0 & \cdots & 1 / n & 0
\end{array}\right) .
$$


Put

$$
h_{n}(z)=z^{n} f_{n}(1 / z)=\sum_{k=0}^{n} \frac{a_{k} z^{k}}{k !} \quad \text { and } \quad \tilde{h}_{n}(z)=z^{n} \tilde{f}_{n}(1 / z) .
$$

Then $z_{k}\left(h_{n}\right)=\frac{1}{z_{k}\left(f_{n}\right)}=\frac{1}{\lambda_{k}\left(F_{n}\right)}=\lambda_{k}\left(F_{n}^{-1}\right)$ and $z_{k}\left(\tilde{h}_{n}\right)=\lambda_{k}\left(\tilde{F}_{n}^{-1}\right)$.

Due to Hurwitz theorem [15, p. 4] if $z_{0}$ is an $m$-fold zero of $h(z)$, then every sufficient small neighborhood of $z_{0}$ contains $m$ zeros counted with their multiplicities of each $h_{n}$ for all sufficiently large $n$. Thus from (3) for all sufficiently large $n$ we have

$$
\min _{k} \Re \lambda_{k}\left(F_{n}^{-1}\right)=\min _{k} \Re z_{k}\left(h_{n}\right)>\gamma .
$$

Lemma 2.1. Let condition (5) hold. Then the spectral radius $r_{s}\left(2 \gamma F_{n}-I\right)$ of the matrix $2 \gamma F_{n}-I$ satisfies the inequality

$$
r_{s}\left(2 \gamma F_{n}-I\right)<1 .
$$

Proof. Let $\mu=x+i y \in \sigma\left(F_{n}^{-1}\right) ; x, y \in \mathbb{R}$. Then $x>\gamma$ and

$$
\begin{aligned}
|2 \gamma / \mu-1|^{2} & =|2 \gamma-\mu|^{2} /|\mu|^{2}=\left((2 \gamma-x)^{2}+y^{2}\right) /|\mu|^{2} \\
& =\left(4 \gamma^{2}-4 \gamma x+x^{2}+y^{2}\right) /|\mu|^{2}<\left(x^{2}+y^{2}\right) /|\mu|^{2}<1 .
\end{aligned}
$$

This proves the lemma.

For an $A \in \mathbb{C}^{n \times n}$ assume that

$$
r_{s}(A)<1
$$

and put

$$
\chi(A):=\sum_{k=0}^{\infty}\left\|A^{k}\right\|^{2}
$$

Note that

$$
\begin{aligned}
\int_{0}^{2 \pi}\left\|\left(I-A e^{i t}\right)^{-1} h\right\|^{2} d t & =\int_{0}^{2 \pi}\left(\sum_{k=0}^{\infty} A^{k} e^{i t k} h, \sum_{j=0}^{\infty} A^{j} e^{i t j} h\right) d t \\
& =2 \pi \sum_{k=0}^{\infty}\left\|A^{k} h\right\|^{2}\left(h \in \mathbb{C}^{n}\right) .
\end{aligned}
$$

Hence, we easily have

$$
\chi(A)=\frac{1}{2 \pi} \int_{0}^{2 \pi}\left\|\left(I-A e^{i t}\right)^{-1}\right\|^{2} d t .
$$

Lemma 2.2. Let $A, \tilde{A} \in \mathbb{C}^{n \times n}$ and condition (6) hold. If, in addition,

$$
\chi(A)\left(\|A-\tilde{A}\|^{2}+2\|A\|\|A-\tilde{A}\|\right)<1,
$$

then $r_{s}(\tilde{A})<1$. 
Proof. Consider the discrete Lyapunov equation

$$
X-A^{*} X A=I,
$$

with given $A \in \mathbb{C}^{n \times n}, X \in \mathbb{C}^{n \times n}$ should be found. It can be directly checked that

$$
X=\sum_{k=0}^{\infty}\left(A^{*}\right)^{k} A^{k}
$$

(see also [12, Lemma 2.1] and references therein). With $C=\tilde{A}-A$ we have

$$
\begin{aligned}
X-\tilde{A}^{*} X \tilde{A} & =X-\left(C^{*}+A^{*}\right) X(C+A) \\
& =X-A^{*} X A-C^{*} X C-A^{*} X C-A^{*} X C \\
& =I-C^{*} X C-A^{*} X C-A^{*} X C .
\end{aligned}
$$

Obviously, $\|X\| \leq \chi(A)$. Thus the inequalities

$$
\|X\|\left(\|C\|^{2}+2\|A\|\|C\|\right) \leq \chi(A)\left(\|C\|^{2}+2\|A\|\|C\|\right)<1
$$

imply that $X-\tilde{A}^{*} X \tilde{A}$ is a positive definite operator and therefore by [7, Theorem 6.1] $r_{s}(\tilde{A})<1$, as claimed.

Lemma 2.3. Let the conditions (5), and

$$
4 \chi\left(2 \gamma F_{n}-I\right)\left(\gamma^{2}\left\|F_{n}-\tilde{F}_{n}\right\|^{2}+\gamma\left\|2 \gamma F_{n}-I\right\|\left\|F_{n}-\tilde{F}_{n}\right\|\right)<1
$$

be fulfilled. Then

$$
\min _{k} \Re \lambda_{k}\left(\tilde{F}_{n}^{-1}\right)>\gamma .
$$

Proof. Put $B=2 \gamma F_{n}-I, \tilde{B}=2 \gamma \tilde{F}_{n}-I$. We have $\tilde{B}-B=2 \gamma\left(\tilde{F}_{n}-F_{n}\right)$. By Lemma 2.1, $r_{s}(B)=r_{s}\left(2 \gamma F_{n}-I\right)<1$. So

$$
\chi(B)=\chi\left(2 \gamma F_{n}-I\right)=\sum_{k=0}^{\infty}\left\|\left(2 \gamma F_{n}-I\right)^{k}\right\|^{2} .
$$

By Lemma 2.2, if conditions (5) and (8) hold, then the inequality $r_{s}\left(2 \gamma \tilde{F}_{n}-\right.$ $I)<1$ is valid. So with $\mu=x+i y \in \sigma\left(\tilde{F}_{n}^{-1}\right)$ we have

$$
\begin{aligned}
1 & >|2 \gamma / \mu-1|^{2}=|2 \gamma-\mu|^{2} /|\mu|^{2}=\left((2 \gamma-x)^{2}+y^{2}\right) /|\mu|^{2} \\
& =\left(4 \gamma^{2}+x^{2}+y^{2}-4 \gamma x\right) /|\mu|^{2}=1+\left(4 \gamma^{2}-4 \gamma x\right) /|\mu|^{2} .
\end{aligned}
$$

Hence,

$$
\left(4 \gamma^{2}-4 \gamma x\right) /|\mu|^{2}<0
$$

and therefore $x>\gamma$, as claimed.

We need the following quantity:

$$
g(A)=\left[N_{2}^{2}(A)-\sum_{k=1}^{n}\left|\lambda_{k}(A)\right|^{2}\right]^{1 / 2} \quad\left(A \in \mathbb{C}^{n \times n}\right),
$$


where $N_{2}(A)=\left(\text { trace } A A^{*}\right)^{1 / 2}$ is the Frobenius (Hilbert-Schmidt norm) of $A$. The following relations are checked in [12, Section 3.1].

$$
g^{2}(A) \leq N_{2}^{2}(A)-\mid \text { Trace } A^{2} \mid \quad \text { and } \quad g^{2}(A) \leq \frac{N_{2}^{2}\left(A-A^{*}\right)}{2}=2 N_{2}^{2}\left(A_{I}\right),
$$

where $A_{I}=\left(A-A^{*}\right) / 2 i$. In addition, $g\left(e^{i t} A+z I\right)=g(A)(z \in \mathbb{C}, t \in \mathbb{R})$. If $A$ is a normal matrix: $A A^{*}=A^{*} A$, then $g(A)=0$. If $A_{1}$ and $A_{2}$ are commuting matrices, then $g\left(A_{1}+A_{2}\right) \leq g\left(A_{1}\right)+g\left(A_{2}\right)$. Moreover, $g^{2}(A) \leq N_{2}^{2}(A)-n(\operatorname{det} A)^{2 / n}$.

According to [12, Theorem 9.5], for any $n \times n$ matrix $A$,

$$
\left\|(A-\lambda I)^{-1}\right\| \leq \sum_{m=0}^{n-1} \frac{g^{m}(A)}{\sqrt{m !} \rho^{m+1}(A, \lambda)}(\lambda \notin \sigma(A)),
$$

where $\rho(A, \lambda):=\min _{k=1, \ldots, n}\left|\lambda-\lambda_{k}(A)\right|$.

Since

$$
g\left(2 \gamma F_{n}-I\right)=g\left(2 \gamma F_{n}\right) \leq N_{2}\left(2 \gamma F_{n}\right)=2 \gamma N_{2}\left(F_{n}\right)
$$

and

$$
\rho\left(2 \gamma F_{n}-I, e^{-i t}\right) \geq 1-r_{s}\left(2 \gamma F_{n}-I\right),
$$

provided that $r_{s}\left(2 \gamma F_{n}-I\right)<1$, according to (9) we have

$$
\left\|\left(I-\left(\gamma F_{n}-I\right) e^{i t}\right)^{-1}\right\| \leq \sum_{m=0}^{n-1} \frac{N_{2}^{m}\left(2 \gamma F_{n}\right)}{\sqrt{m !}\left(1-r_{s}\left(2 \gamma F_{n}-I\right)\right)^{m+1}} .
$$

Hence, (7) implies

$$
\begin{aligned}
\chi\left(2 \gamma F_{n}-I\right) & =\frac{1}{2 \pi} \int_{0}^{2 \pi}\left\|\left(I-\left(\gamma F_{n}-I\right) e^{i t}\right)^{-1}\right\|^{2} d t \\
& \leq\left(\sum_{m=0}^{n-1} \frac{N_{2}^{m}\left(2 \gamma F_{n}\right)}{\sqrt{m !}\left(1-r_{s}\left(2 \gamma F_{n}-I\right)\right)^{m+1}}\right)^{2} .
\end{aligned}
$$

Note that

$$
N_{2}^{2}\left(F_{n}\right)=\sum_{k=1}^{n}\left(\left|a_{k}\right|^{2}+\frac{1}{k^{2}}\right)-1 \leq \sum_{k=1}^{\infty}\left|a_{n}\right|^{2}+\zeta(2)-1=\psi^{2}(h)
$$

and

$$
\begin{aligned}
r_{s}\left(2 \gamma F_{n}-I\right) & =\max _{k=1, \ldots, n}\left|2 \gamma \lambda_{k}\left(F_{n}\right)-1\right|=\max _{k=1, \ldots, n}\left|\frac{2 \gamma}{z_{k}\left(h_{n}\right)}-1\right| \\
& \leq \sup _{k=1,2, \ldots}\left|\frac{2 \gamma}{z_{k}(h)+\epsilon_{n}}-1\right|,
\end{aligned}
$$

where $\epsilon_{n} \rightarrow 0$ as $n \rightarrow \infty$. 
Thus, for sufficiently large $n$,

$$
\begin{aligned}
\chi\left(2 \gamma F_{n}-I\right) & \leq\left(\sum_{m=0}^{n-1} \frac{(2 \gamma \psi(h))^{m}}{\sqrt{m !}\left(1-\sup _{k=1,2, \ldots}\left|\frac{2 \gamma}{z_{k}(h)+\epsilon_{n}}-1\right|\right)^{m+1}}\right)^{2} \\
& \leq\left(\sum_{m=0}^{n-1} \frac{(2 \gamma \psi(h))^{m}}{\sqrt{m !}\left(1-\sup _{k=1,2, \ldots}\left|\frac{2 \gamma}{z_{k}(h)}-1\right|\right)^{m+1}}\right)^{2}+\hat{\epsilon}_{n} \\
& \leq \xi(h, \gamma)+\hat{\epsilon}_{n},
\end{aligned}
$$

where $\hat{\epsilon}_{n} \geq 0$ and $\hat{\epsilon}_{n} \rightarrow 0$ as $n \rightarrow \infty$. In addition,

$$
\left\|2 \gamma F_{n}-I\right\| \leq 1+2 \gamma\left\|F_{n}\right\| \leq 1+2 \gamma N_{2}\left(F_{n}\right) \leq 1+2 \gamma \psi(h) .
$$

Moreover,

$$
\left\|F_{n}-\tilde{F}_{n}\right\|^{2} \leq \sum_{k=1}^{\infty}\left|a_{k}-\tilde{a}_{k}\right|^{2}=q^{2} .
$$

So, condition (8) is provided by the inequality

$$
4\left(\xi(h, \gamma)+\hat{\epsilon}_{n}\right)\left(\gamma^{2} q^{2}+\gamma q(1+2 \gamma \psi(h))<1 .\right.
$$

By Lemma 2.3, for sufficiently large $n$ we have

$$
\min _{k} \Re z_{k}\left(\tilde{h}_{n}\right)=\min _{k} \Re \lambda_{k}\left(\tilde{F}_{n}^{-1}\right)>\gamma .
$$

Now letting $n \rightarrow \infty$, we get the required result.

\section{Perturbed polynomials}

In this section we considerably simplify Theorem 1.1 in the case of the polynomials

$$
p(z)=\sum_{k=0}^{n} b_{k} z^{n-k} \quad \text { and } \quad \tilde{p}(z)=\sum_{k=0}^{n} \tilde{b}_{k} z^{n-k}
$$

$\left(b_{0}=\tilde{b}_{0}=1 ; b_{k}, \tilde{b}_{k} \in \mathbb{C}, k=1, \ldots, n\right)$. The theory of polynomials in spite its long history cf. $[1,16]$ continues to attract an attention of many mathematicians, for example see $[14,18,19]$. However to the best of our knowledge the above pointed problem has not been considered even for polynomials.

Assume that

$$
\hat{\beta}(p):=\min _{k=1, \ldots, n} \Re z_{k}(p)>0
$$

and put

$$
\hat{q}_{n}:=\left(\sum_{k=1}^{\infty}\left|b_{k}-\tilde{b}_{k}\right|^{2}\right)^{1 / 2} \quad \text { and } \quad \eta(p):=\sum_{j, k=0}^{n-1} \frac{(k+j) ! \hat{\psi}_{n}^{k+j}(p)}{2^{k+j} \hat{\beta}^{k+j+1}(p)(k ! j !)^{1 / 2}},
$$


where

$$
\hat{\psi}_{n}(p)=\left(n-1+\sum_{k=1}^{n}\left|b_{k}\right|^{2}\right)^{1 / 2} .
$$

Theorem 3.1. Let the conditions (10) and

$$
\hat{q}_{n} \eta(p)<1
$$

hold. Then

$$
\min _{k=1,2, \ldots} \Re z_{k}(\tilde{p})>0 .
$$

Proof. Note that $p$ and $\tilde{p}$ are the characteristic polynomials of the matrices

$$
C=\left(\begin{array}{ccccc}
-b_{1} & -b_{2} & \cdots & -b_{n-1} & -b_{n} \\
1 & 0 & \cdots & 0 & 0 \\
0 & 1 & \cdots & 0 & 0 \\
\vdots & \vdots & \cdots & \vdots & \vdots \\
0 & 0 & \ddots & 1 & 0
\end{array}\right)
$$

and

$$
\tilde{C}=\left(\begin{array}{ccccc}
-\tilde{b}_{1} & -\tilde{b}_{2} & \cdots & -\tilde{b}_{n-1} & -\tilde{b}_{n} \\
1 & 0 & \cdots & 0 & 0 \\
0 & 1 & \cdots & 0 & 0 \\
\vdots & \vdots & \ddots & \vdots & \vdots \\
0 & 0 & \cdots & 1 & 0
\end{array}\right)
$$

respectively. So

$$
\beta(C):=\min _{k} \Re \lambda_{k}(C)=\hat{\beta}(p)>0 .
$$

As is well known [4, Section 1.5], if condition (13) is fulfilled, then the matrix Lyapunov equation

$$
C^{*} Y+Y C=2 I,
$$

has a selfadjoint solution $Y$. Due to Lemma 3.1 from [13], under the condition

$$
\|Y\|\|\tilde{C}-C\|<1
$$

one has $\min _{k=1, \ldots, n} \Re \lambda_{k}(\tilde{C})>0$ and, therefore, (12) is valid. Put

$$
\hat{\eta}(C):=\sum_{j, k=0}^{n-1} \frac{(k+j) ! g^{k+j}(C)}{2^{k+j} \beta^{k+j+1}(p)(k ! j !)^{1 / 2}} .
$$

Due to Lemma 1.9.2 from [10] $\|Y\| \leq \hat{\eta}(C)$. But,

$$
g^{2}(C) \leq N_{2}^{2}(C)=n-1+\sum_{k=1}^{n}\left|b_{k}\right|^{2}=\hat{\psi}_{n}^{2}(p) .
$$


Thus, $\hat{\eta}(C)=\eta(p)$. Since $\|\tilde{C}-C\|=\hat{q}_{n}$, condition (14) is provided by condition (11). This proves the theorem.

\section{Estimates FOR $\xi(h, \gamma), q$ AND $\psi(h)$}

By the Schwarz inequality for constants $c \in(0,1)$ and $a \geq 0$ we have

$$
\sum_{k=0}^{\infty} \frac{a^{k}}{\sqrt{k !}}=\sum_{k=0}^{\infty} \frac{(a c)^{k}}{c^{k} \sqrt{k !}} \leq\left(\sum_{j=0}^{\infty} c^{2 j} \sum_{k=0}^{\infty} \frac{a^{2 k}}{c^{2 k} k !}\right)^{1 / 2}=\frac{1}{\left(1-c^{2}\right)^{1 / 2}} e^{a^{2} /\left(2 c^{2}\right)} .
$$

Thus

$$
\xi(h, \gamma) \leq \frac{1}{r^{2}(h, \gamma)\left(1-c^{2}\right)} \exp \left[\frac{4(\gamma \psi(h))^{2}}{r^{2}(h, \gamma) c^{2}}\right] .
$$

In particular, taking $c^{2}=1 / 2$ we obtain

$$
\xi(h, \gamma) \leq \frac{2}{r^{2}(h, \gamma)} \exp \left[\frac{8(\gamma \psi(h))^{2}}{r^{2}(h, \gamma)}\right]
$$

If

$$
\left|a_{k}\right| \leq \text { const } c^{k}(c \in(0,1), k=1,2, \ldots),
$$

then for $\Re z \geq 1$ function $h$ admits the Laplace transform

$$
H(z)=(\mathcal{L} h)(z)=\int_{0}^{\infty} e^{-z t} h(t) d t=\sum_{k=0}^{\infty} \frac{a_{k}}{z^{k+1}},
$$

which can be extended to $|z| \geq 1$. Thus

$$
\left\|H\left(e^{i t}\right)\right\|_{L^{2}(0,2 \pi)}:=\left[\frac{1}{2 \pi} \int_{0}^{2 \pi} H\left(e^{i t}\right) d t\right]^{1 / 2}=\left[\sum_{k=0}^{\infty}\left|a_{k}\right|^{2}\right]^{1 / 2}
$$

and, therefore,

$$
\text { (16) } \psi(h)=\left(\left\|H\left(e^{i t}\right)\right\|_{L^{2}(0,2 \pi)}^{2}+\zeta(2)-2\right)^{1 / 2} \leq\left(\max _{|z|=1} \mid H(z) \|^{2}+\zeta(2)-2\right)^{1 / 2} \text {. }
$$

Since $\zeta(2) \leq 1.645$, we have

$$
\psi(h) \leq\left(\left\|H\left(e^{i t}\right)\right\|_{L^{2}(0,2 \pi)}^{2}-0.355\right)^{1 / 2} \leq\left(\max _{|z|=1}|H(z)|^{2}-0.355\right)^{1 / 2} .
$$

Similarly,

$$
q:=\left\|H\left(e^{i t}\right)-\tilde{H}\left(e^{i t}\right)\right\|_{L^{2}(0,2 \pi)} \leq \max _{|z|=1}\left|H\left(e^{i t}\right)-\tilde{H}\left(e^{i t}\right)\right|,
$$

where $\tilde{H}(z)$ the Laplace transform to $\tilde{h}$. 


\section{EXAMPLE}

Consider the functions $h(z)=(1-z) e^{0.1 z}$ and $\tilde{h}(z)=(1-a z) e^{0.1 z}$, where $a$ is a positive constant. $h(z)$ has a unique root $z_{1}(h)=1$. So condition (3) holds for example, for $\gamma=0.5$. Clearly, condition (4) holds if $a<2$. Let us compare this result with Theorem 1.1. To this end note that

$$
H(z)=\left[\mathcal{L}\left(e^{0.1 t}-t e^{0.1 t}\right)\right](z)=\frac{1}{z-0.1}-\frac{1}{(z-0.1)^{2}} .
$$

Hence, $\max _{|z|=1}|H(z)| \leq 2.346$. Due to $(16) \psi(h)<2$. Besides,

$$
\sup _{k}\left|\frac{2 \gamma}{z_{k}(h)}-1\right|=\left|\frac{2 \cdot 1 / 2}{1}-1\right|=0,
$$

and therefore $r(h, 0.5)=1$. Hence, by $(15)$

$$
\xi(h, \gamma) \leq 2 \exp \left[8(0.5 \cdot 2)^{2}\right]=2 e^{8} .
$$

In addition,

Hence, by (17)

$$
\tilde{H}(z)-H(z)=(1-a) \frac{1}{(z-0.1)^{2}}
$$

$$
q \leq|a-1| \max _{|z|=1} \frac{1}{|z-0.1|^{2}} \leq|a-1| 1.235 .
$$

So, by Theorem 1.1, inequality (4) holds with $\gamma=0.5$, provided that

$$
\begin{gathered}
8 e^{8}\left((0.5 \cdot 1.235|a-1|)^{2}+0.5 \cdot 1.235|a-1|(1+4 \cdot 0.5)\right)= \\
=8 e^{8}\left(0.381|a-1|^{2}+1.854|a-1|\right)<1 .
\end{gathered}
$$

\section{REFERENCES}

[1] P. Borwein, T. Erdelyi, Polynomials and Polynomial Inequalities, Springer-Verlag, New York, 1995.

[2] J. G. Clunie, A. Edrei, Zeros of successive derivatives of analytic functions having a single essential singularity II, J. Anal. Math., 56 (1991), 141-185.

[3] T. Craven, G. Csordas, W. Smith, Zeros of derivatives of entire functions, Proc. Amer. Math. Soc., 101 (1987), 323-326.

[4] Yu. L. Daleckiı̌, M.G. Kreĭn, Stability of Solutions of Differential Equations in Banach Space, Amer. Math. Soc., Providence, R. I, 1971.

[5] A. Edrei, E. B. Saff, R. S. Varga, Zeros of Sections of Power Series, Lecture Notes in Math., 1002, Springer Verlag, Berlin, 1983.

[6] S. Edwards, S. Hellerstein, Non-real zeros of derivatives of real entire functions and the Pólya-Wiman conjectures, Complex Variables, Theory Appl., 47 (1) (2002), 2557.

[7] T. Eisner, Stability of Operators and Operator Semigroups, Operator Theory: Advances and Applications, 209, Birkhäuser Verlag, Basel, 2010. 
[8] R. M. Genthner, On the zeros of the derivatives of some entire functions of finite order, Proc. Edinb. Math. Soc., 28 (3) (1985), 381-407.

[9] M. I. Gil', Perturbations of zeros of a class of entire functions, Complex Variables, Theory Appl., 42 (2000), 97-106.

[10] M. I. Gil', Explicit Stability Conditions for Continuous Systems, Lectures Notes In Control and Information sciences, 314, Springer Verlag, 2005.

[11] M. I. Gil', Localization and Perturbation of Zeros of Entire Functions, Lecture Notes in Pure and Applied Mathematics, 258, CRC Press, Boca Raton, FL, 2010.

[12] M. I. Gil', Operator Functions and Operator Equations, World Scientific, New Jersey, 2018.

[13] M. I. Gil', Norm estimates for function Lyapunov equations and applications, J. Franklin Inst., 355 (10) (2018), 4241-4247.

[14] M. H. Gulzar, On the location of zeros of a polynomial, Anal. Theory Appl., 28 (3) (2012), 242-247.

[15] M. Marden, Geometry of Polynomials, Amer. Math. Soc., Providence, R.I., 1985.

[16] G. V. Milovanović, D. S. Mitrinović, Th. M. Rassias, Topics in Polynomials: Extremal Problems, Inequalities, Zeros, World Scientific Publ., Singapore, 1994.

[17] P. C. Rosenbloom, Perturbation of zeros of analytic functions. I., Journal of Approximation Theory, 2 (1969), 111-126.

[18] D. M. Simeunović, On the location of zeros of some polynomials, Mathematica Moravica, 16 (2) (2012), 59-61.

[19] H. A. Soleiman Mezerji, M. Bidkham, Cauchy type results concerning location of zeros of polynomials, Acta Math. Univ. Comenian. (N.S.), 83 (2) (2014), 267-279.

Michael Gil'

Department of Mathematics

Ben Gurion University of the Negev

P.O. Box 653, BeER-Sheva 84105

ISRAEL

E-mail address: gilmi@bezeqint.net 